\title{
Magnetic Proximity Coupling of Quantum Emitters in WSe to van der Waals Ferromagnets
}

\author{
Kamran Shayan ${ }^{1,2}, \mathrm{Na} \mathrm{Liu}^{1,2}$, Andrew $\mathrm{Cupo}^{3}$, Yichen $\mathrm{Ma}^{1,2}$, Yue Luo ${ }^{1,2}$, Vincent Meunier ${ }^{3}$ and Stefan \\ Strauf ${ }^{1,2^{*}}$ \\ ${ }^{1}$ Department of Physics, Stevens Institute of Technology, Hoboken, NJ 07030, USA \\ ${ }^{2}$ Center for Quantum Science and Engineering, Stevens Institute of Technology, Hoboken, NJ 07030, \\ USA. \\ ${ }^{3}$ Department of Physics, Applied Physics, and Astronomy, Rensselaer Polytechnic Institute, Troy, NY \\ 12180, USA. \\ *Email: strauf@stevens.edu
}

\section{Supplementary Note 1. Magneto-PL properties of quantum emitters in the flux-grown versus CVT-grown crystals of WSe2.}

Here we compare magneto-optical properties of quantum emitters generated in $\mathrm{WSe}_{2}$ monolayers exfoliated from flux-grown crystals with those of $\mathrm{WSe}_{2}$ monolayers exfoliated from CVT-grown crystals. Particularly, the flux-grown material has some very attractive optical properties including rather low point defect densities ${ }^{1}$ that gives rise to record high quantum yields of $16.5 \%$ in the bare as well as $65 \%$ when coupled to a nanocavity mode ${ }^{2}$, and further enables quantum light emission up to $160 \mathrm{~K} .{ }^{3}$ We determined the fine structure splitting $\Delta_{0}$ of 22 quantum emitters from fluxgrown and 26 quantum emitters from CVT-grown monolayers, respectively. Statistical studies in Figure S1a show that the mean values for $\Delta_{0}$ for the flux-grown case are with $0.47 \pm 0.11$ significantly smaller as compared to CVT-grown monolayers with values of $0.7 \pm 0.15$. Since the origin of $\Delta_{0}$ is anisotropic strain in the basal plane of $\mathrm{WSe}_{2},{ }^{4}$ it is apparent that the lower defect density in the flux-grown material gives rise to reduction of the anisotropic strain in the lattice. In addition, strain-induced quantum emitters in flux-grown material are characterized by somewhat smaller 0D exciton g-factors with mean values of 5.4 \pm 1.1 , while quantum emitters in CVT-grown material display average values of $7.5 \pm 1.8$. These findings highlight the need to study magneticproximity effect for the same quantum emitter, as demonstrated in the main manuscript, since the magneto-optical properties not only depend on the local strain but also on TMDC material growth conditions. To eliminate some of these variations in the exciton g-factor of strain-induced quantum emitters, we present in the main manuscript solely data from CVT -grown material. Note that our technique to determine properties of the same quantum emitter before and after coupling, as described in the second half of the manuscript, is an excellent way to eliminate variations in the (largely unknown) microscopic origin of the exciton g-factor. 
(a)

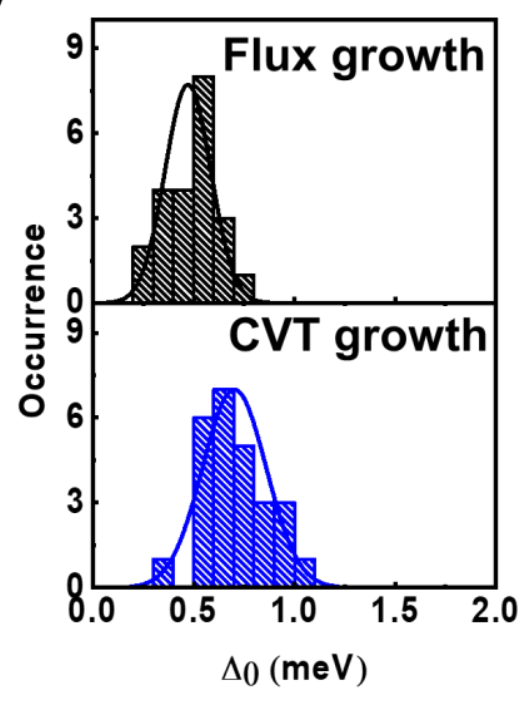

(b)

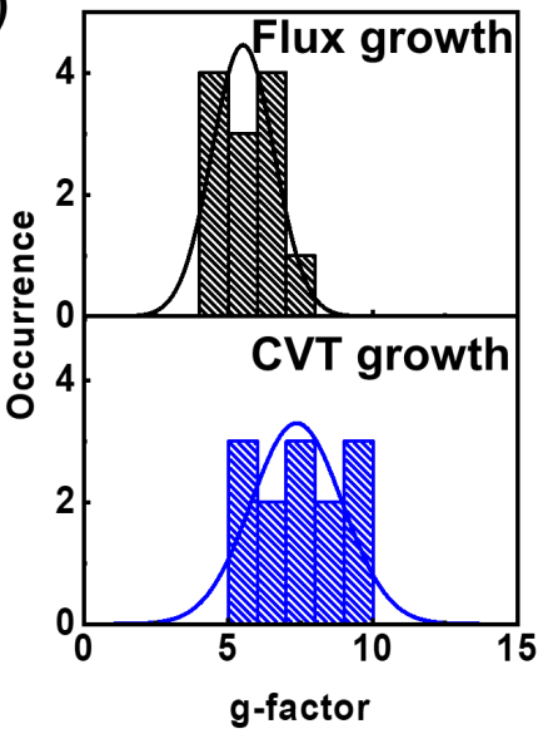

Figure S1: Occurrence histograms of fine-structure splitting values $\Delta_{0}$ (a) and $0 \mathrm{D}$ exciton g-factors (b) comparing monolayers exfoliated from CVT-grown crystals (blue) and flux-grown crystals (black).

\section{Supplementary Note 2. Site-controlled quantum emitters in CGT/ WSe2 heterostructure}

To quantify of the magneto-PL properties of site-controlled quantum emitters, we have prepared wafers with gold stressors that are able to deterministically induce quantum emitters into a precise location. Nanopillars are fabricated on silicon substrate by electron beam lithography as was detailed in Ref. 3. Subsequently, CGT multilayers are mechanically exfoliated and transferred onto the nanopillars followed by stamping a monolayer of $\mathrm{WSe}_{2}$ over the CGT layer. The interface quality between layers was improved by using our established annealing technique ${ }^{5}$ to achieve a clean interface free from possible residual contamination of the dry transfer process. Figure S2 shows a characteristic quantum emitter in this layering sequence characterized by a $0 \mathrm{D}$ exciton $\mathrm{g}$ factor of $11.2 \pm 0.2$ and CGT saturation magnetization around $6.5 \mathrm{~T}$. The g-factor is significantly larger than the average values found in the uncoupled case and thus demonstrates proximity coupling of site-controlled quantum emitters. The layer ordering however does not allow precise determination of the MEF splitting energy. Note that the saturation point of this quantum emitter is slightly higher as compared to other cases, possible related to the exact local strain profile that can cause a change of the CGT magnetization direction from the $c$-axis to the $a b$-plane ${ }^{6}$. It might be that in this case that the CGT remains somewhat tilted over in the nanopillar, causing the external field to be misaligned with the magnetization direction of CGT. 

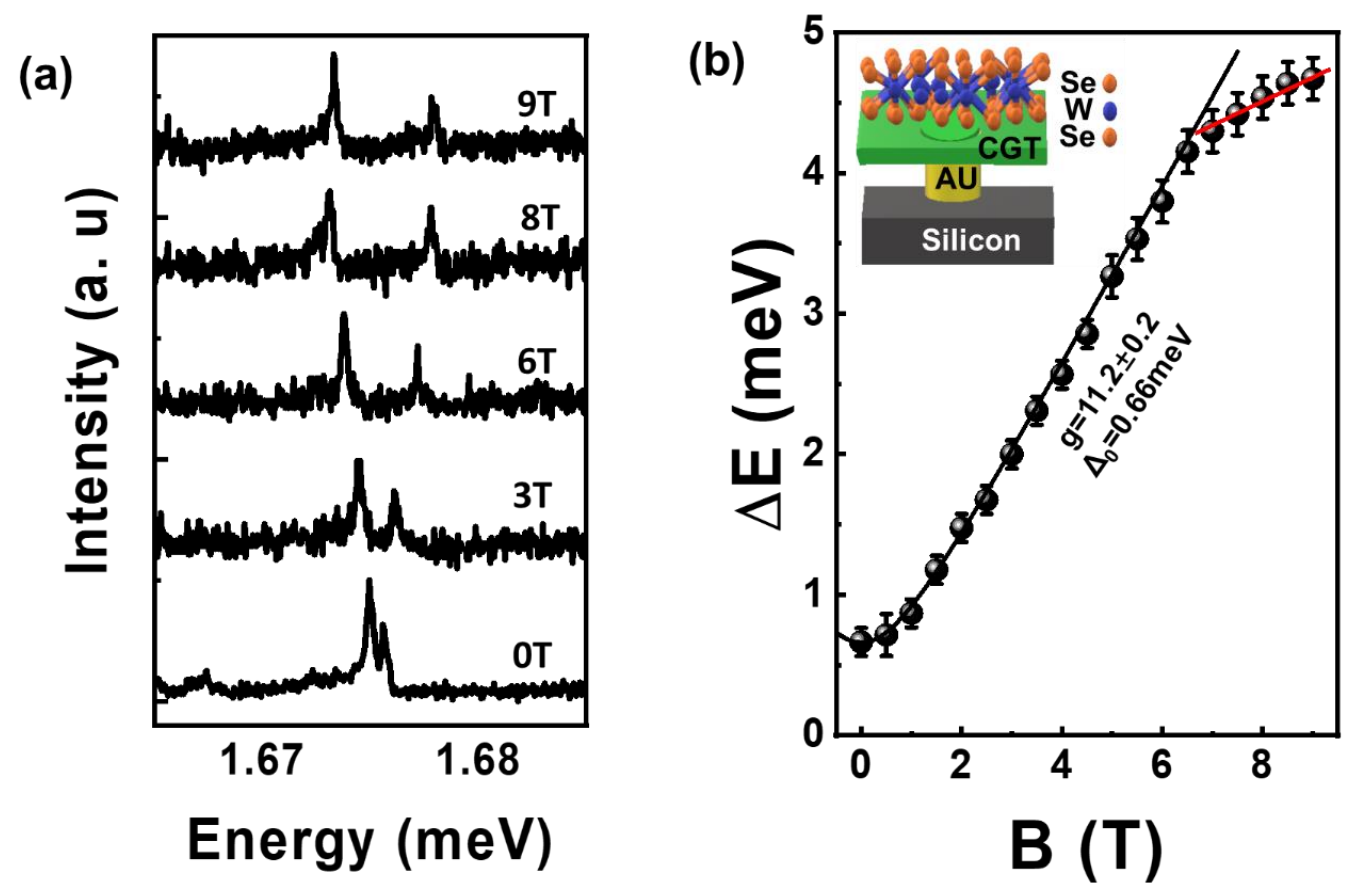

Figure S2: Magneto-optical characterization of site-controlled quantum emitters in CGT/WSe 2 heterostructure ordering. (a) Magnetic field dependence of exemplary photoluminescence spectra recorded in Faraday geometry. (b) Corresponding Zeeman energy splitting $\Delta \mathrm{E}$ as function of magnetic field strength showing a $\mathrm{g}$ factor of $\mathrm{g}=11.2 \pm 0.2$ and a fine structure splitting $\Delta_{0}=0.66$ meV. Inset: schematic of WSe $/$ CGT heterostructure strained over pillars. This case utilized CVTgrown material. All data recorded at $4 \mathrm{~K}$.

\section{Supplementary Note 3. Site-controlled quantum emitters in WSe/CGT heterostructures}

Here we provide additional magneto-PL studies of quantum emitters for the alternative layer sequence with $\mathrm{WSe}_{2}$ transferred onto the nanopillar arrays followed by CGT on top with optical measurements carried out through the ferromagnetic layer that remains partially transparent. Figures S3a and S4a show the zero-field spectrum for two strain-induced quantum emitters before and after coupling to the CGT layer. Spectra before (after) coupling are integrating for 1s (20s). As a key finding, the fine structure splitting in Figure S3a (Figure S4a) remains with $\Delta_{0}=400 \pm 10$ $\mu \mathrm{eV}\left(\Delta_{0}=570 \pm 10 \mu \mathrm{eV}\right)$ before and $\Delta_{0}=410 \pm 10 \mu \mathrm{eV}\left(\Delta_{0}=590 \pm 10 \mu \mathrm{eV}\right)$ after coupling unchanged within the error bar, clearly indicating that the degree of strain anisotropy is not affected by the stamping process of another layer on top of the $\mathrm{WSe}_{2}$ residing on the nanopillar arrays. Since CGT displays soft ferromagnetic behavior, i.e. and absence of ferromagnetism without an applied external magnetic field, no effect from the ferromagnetic proximity coupling is expected in the zero-field case. From the magnetic field studies in Figure S3b and Figure $\mathbf{S 4 b}$ one can extract a proximity enhanced g-factor that is $1.66 \pm 0.3$ and $1.4 \pm 0.1$ times larger, respectively, as compared to the uncoupled case, in close agreement with the average in ensemble studies. Both quantum emitters show saturation magnetization of CGT at fields of 5.5 T in Figure S3b and 3.5 T in Figure S4b. The corresponding magnetic exchange field (MEF) splitting values were extracted from the 
energy difference between the coupled and uncoupled case, resulting in $1.2 \pm 0.01 \mathrm{meV}$ in Figure $\mathrm{S} 3 \mathrm{~b}$ and significantly reduced value of $0.5 \pm 0.01 \mathrm{meV}$ for Figure S4b.
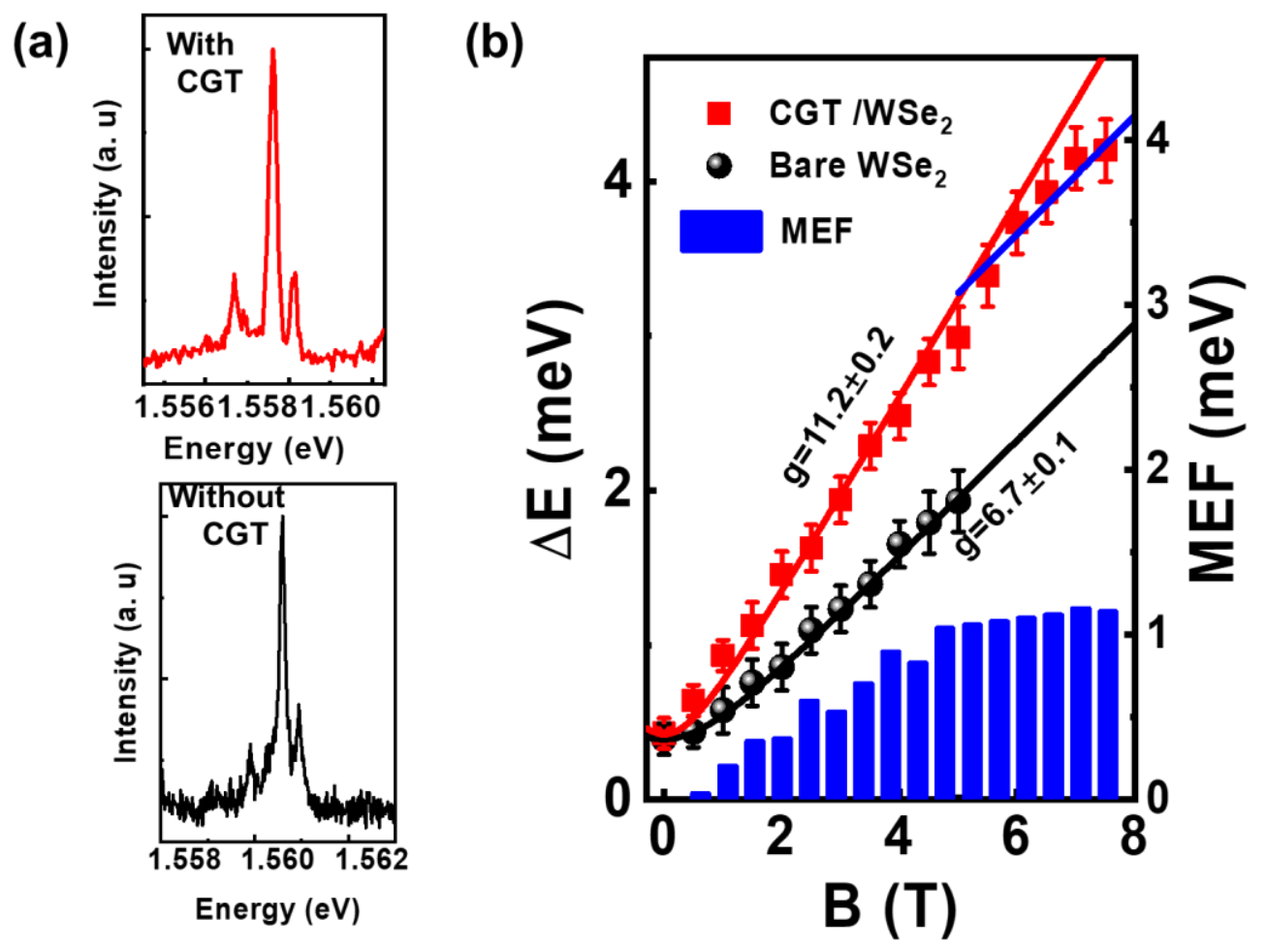

Figure S3. Magneto-optical characterization of quantum emitters in WSe $2 / C G T$ heterostructures. (a) PL spectra for the Zeeman doublet of an individual quantum emitter recorded at 0T without (bottom) and with CGT attached (top) to form the heterostructure. (b) Zeeman splitting $\Delta \mathrm{E}$ as a function of magnetic field before (black dots) and after proximity coupling (red dots). The solid lines black and red lines follow the standard equation to extract the g-factor. The blue solid line fits a linear trend in the saturation regime. All data recorded at $4 \mathrm{~K}$. 

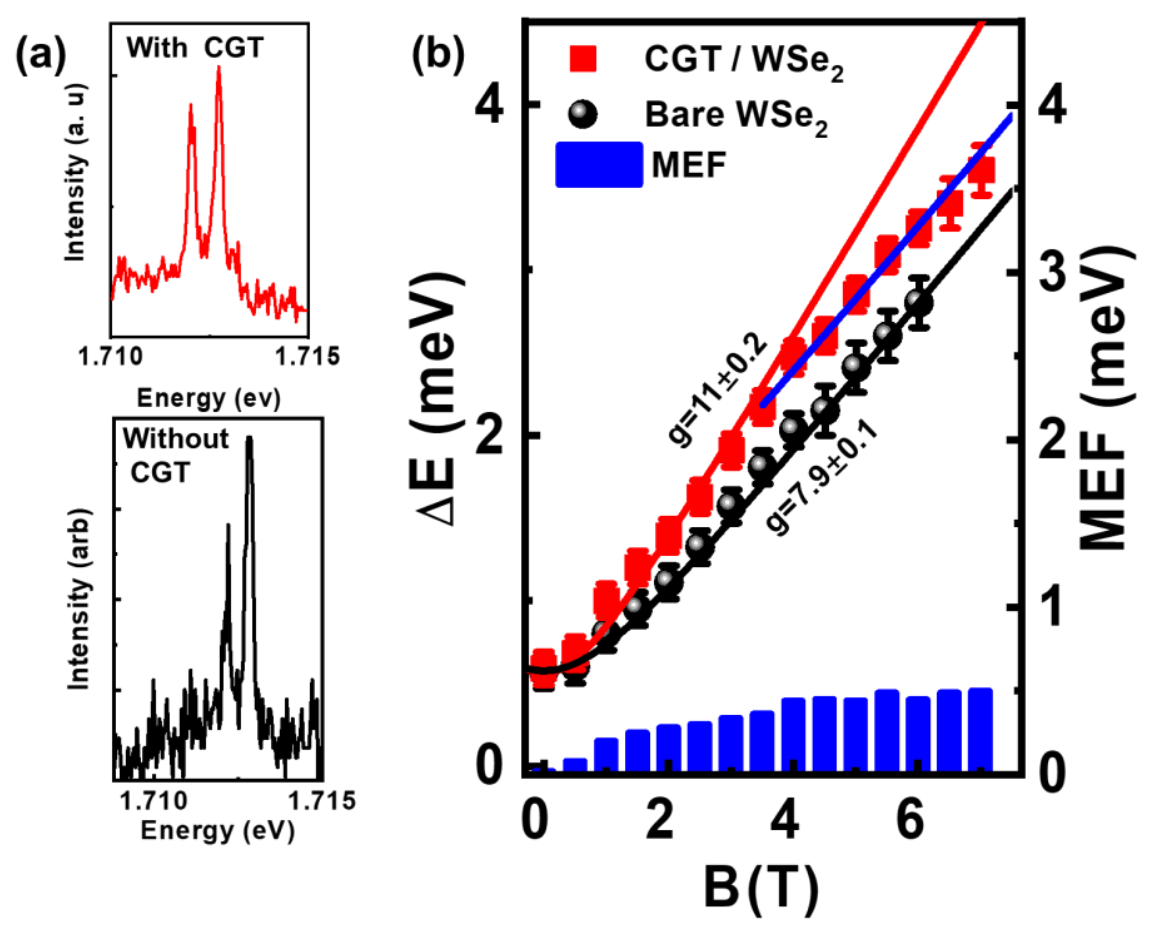

Figure S4. Magneto-optical characterization of quantum emitters in $\mathrm{WSe}_{2} / \mathrm{CGT}$ heterostructures. (a) PL spectra for the Zeeman doublet of an individual quantum emitter recorded at 0T without (bottom) and with CGT attached (top) to form the heterostructure. (b) Zeeman splitting $\Delta \mathrm{E}$ as a function of magnetic field before (black dots) and after proximity coupling (red dots). The solid lines black and red lines follow the standard equation to extract the g-factor. The blue solid line fits a linear trend in the saturation regime. All data recorded at $4 \mathrm{~K}$. 


\section{Reference:}

[1] Edelberg, D.; Rhodes, D.; Kerelsky, A; Kim, B.; Wang, J.; Zangiabadi, A.; and Kim, C.; Abhinandan, A.; Ardelean, J.; Scully, M.; Scullion, D.; Embon, L.; Zu, R.; Santos, J. G. E.; Balicas, L.; Marianetti, C.; Barmak, K.; Zhu, X.; Hone, J.; Pasupathy, A. N. Approaching the Intrinsic Limit in Transition Metal Diselenides via Point Defect Control. Nano Lett, 2019, 19 (7), 4371-4379.

[2] Luo, Y.; Shepard, G. D.; Ardelean, J. V.; Rhodes, D. A.; Kim, B.; Barmak, K.; Hone, J. C.; Strauf, S. Deterministic Coupling of Site-Controlled Quantum Emitters in Monolayer WSe 2 to Plasmonic Nanocavities. Nat. Nanotechnol, 2018, 13 (12), 1137-1142.

[3] Luo, Y.; Liu, N.; Li, X.; Hone, J. C.; Strauf, S. Single Photon Emission in WSe 2 Up 160 K by Quantum Yield Control. 2D Mater, 2019, 6 (3), 035017.

[4] Kumar, S.; Brotóns-Gisbert, M.; Al-Khuzheyri, R.; Branny, A.; Ballesteros-Garcia, G.; Sánchez-Royo, J. F.;Gerardot, B. D. Resonant Laser Spectroscopy of Localized Excitons in Monolayer WSe2. Optica, 2016, 3 (8), 882-886.

[5] Shepard, G. D.; Ajayi, O. A.; Li, X.; Zhu, X. Y.; Hone, J.; Strauf, S. Nanobubble Induced Formation of Quantum Emitters in Monolayer Semiconductors. 2D Mater, 2017,4 (2), 021019.

[6] Lin, Z.; Lohmann, M.; Zulfikhar, A. A.; Tang, C.; Li, J.; Xing, W.; Zhong, J.; Jia, S.; Han, W.; Coh, S.; Beyermann, W.; Shi, J. Pressure-induced Spin Reorientation Transition in Layered Ferromagnetic Insulator $\mathrm{Cr}_{2} \mathrm{Ge}_{2} \mathrm{Te}_{6}$. PR Materials, 2018, 2 (5), 051004. 